\title{
Assessing the safety of physician-directed nurse-administered propofol sedation in low-risk patients undergoing endoscopy and colonoscopy
}

\section{(9)(1) $\odot$}

\author{
Authors \\ Dharshan Sathananthan ${ }^{1}$, Edward Young ${ }^{2}$, Garry Nind ${ }^{3}$, Biju \\ George $^{3}$, Angelie Ashby ${ }^{4}$, Sharon Drummond ${ }^{4}$, Kasia Redel ${ }^{4}$, \\ Neville Green ${ }^{5}$, Rajvinder Singh ${ }^{3}$ \\ Institutions \\ 1 Royal Adelaide Hospital - Gastroenerology, Adelaide, South \\ Australia \\ 2 University of Adelaide Medical School, Adelaide, South Australia \\ 3 Lyell McEwin Hospital - Gastroenterology, Adelaide, South \\ Australia \\ 4 University of Adelaide/Lyell McEwin Hospital, Endoscopy Unit, \\ Elizabeth Vale, South Australia \\ 5 Lyell McEwin Hospital - Anaesthetics, Elizabeth Vale, South \\ Australia
}

submitted 15.6.2016

accepted after revision 2.11.2016

\section{Bibliography}

DOI http://dx.doi.org/10.1055/s-0042-121667 |

Endoscopy International Open 2017; 05: EE110-EE115

(c) Georg Thieme Verlag KG Stuttgart · New York

ISSN 2364-3722
Corresponding author

Dharshan Sathananthan, Royal Adelaide Hospital -

Gastroenterology, North Terrace Adelaide, South Australia 5000,

Australia

Fax: +83384860

dharshan.satha@gmail.com

\section{ABSTRACT}

Background and study aims Physician-directed nurse-administered balanced propofol sedation (PhD NAPS) in patients undergoing endoscopy and/or colonoscopy is being increasingly utilized worldwide. However, this method of sedation is not universally employed in Australian hospitals due to concerns surrounding its safety. The aim of this study was to assess the safety of PhD NAPS in lowrisk patients undergoing endoscopy and/or colonoscopy.

Patients and methods This study was conducted at a single tertiary teaching hospital in Adelaide, Australia. It was a prospective study involving 1000 patients with an ASA score of 1-3 presenting with any indication for endoscopy, colonoscopy or both. A total of 981 patients (451 male) with a mean age of 53 years (range: 16 87) were recruited from January 2010 to October 2012. 440 endoscopies, 420 colonoscopies, and 121 combined procedures were performed. The intra-procedural adverse events (AEs) were recorded.

Results There were no major intra-procedural adverse events. Minor AEs occurred in $6.42 \%$ of patients, and resolved spontaneously or with intravenous fluid boluses in all cases.

Conclusion PhD NAPS is safe when the proceduralist and nursing staff are adequately trained and strict patient selection criteria are used.

\section{Introduction}

Propofol is an ultra-short acting sedative agent with amnestic effects [1]. Its use in gastroenterological procedures such as gastroscopy and colonoscopy is favoured due to its rapid onset and offset of action. It is also associated with fast recovery times and high patient- and physician-reported satisfaction [2]. Despite these appreciable advantages, propofol sedation is not without its drawbacks. It has a narrow therapeutic index and can cause irreversible respiratory depression - the most feared adverse reaction. As a result it has traditionally been administered by anesthetists. However, trained nurses are now administering propofol sedation throughout the world, as it likely confers a significant economic benefit. As of 2008, over 460000 patients worldwide have undergone endoscopist-administered propofol sedation [3]. Despite these numbers, and numerous studies demonstrating the safety of this sedative agent in gastrointestinal procedures [2,4-12), concerns about the safety of the agent being administered by non-anesthetists have been repeatedly voiced [13]. Most studies regarding nonanesthetist administered Propofol sedation have been hampered by either the retrospective nature of the study, small numbers or lack of stringent patient selection criteria. We hence embarked on a prospective study to evaluate the safety of Physician Directed Nurse Administered Propofol Sedation (PhD NAPS) in low-risk American Society of Anaesthesiologists (ASA) I, II and suitable ASA III patients using strict criteria presenting for an endoscopy, colonoscopy or both procedures. 


\section{Patients and methods}

\section{Study population}

The local institutional ethics review panel approved the study. Outpatients and stable inpatients with an ASA score of I, II and III presenting with any indication for endoscopy or colonoscopy or both were prospectively recruited between January 2010 and October 2012. All study participants were aged 18 years or older and provided informed consent. Patients deemed suitable by the endoscopist for PhD NAPS were referred to a nurse sedationist clinic before undergoing their scheduled procedure. During this appointment, the patient's ASA scores were calculated and their suitability to undergo PhD NAPS was determined using a detailed questionnaire concentrating on their past medical history and any anesthetic complications from previous surgeries. Where there was doubt surrounding the patient's safety to undergo PhD NAPS, an anesthetic review was sought. All patients with an ASA score of III underwent review by an anesthetist who decided which patients were suitably low-risk for PhD NAPs.

Patients with a BMI greater than 35, previous complications of anesthesia, a potentially difficult airway, planned endotracheal intubation and/or a Mallampati score of 4 were excluded from this study. We believe this to be an accurate representation of the stringent selection criteria that should be employed if PhD NAPS is to be used in the future.

\section{Study design and sedation protocol}

The primary goal was to assess the safety of PhD NAPs in lowrisk patients. "Balanced" in this context refers to the use of propofol in conjunction with midazolam, as opposed to the use of propofol as a sole agent. This practice, which has been the mainstay of how PhD NAPS has been administered in our institution over the preceding years, was utilized to reduce the overall propofol sedation requirement during the procedure. The procedural duration, recovery time, intra- and post-procedural complications as well the patient's safety were analyzed.

Prior to each procedure, the ASA score of the patient was recalculated. A single peripheral intravenous (IV) line was inserted. Before induction of sedation, blood pressure (BP), heart rate $(\mathrm{HR})$ and oxygen saturation $\left(\mathrm{SaO}_{2}\right)$ were measured. BP was measured throughout at 2-minute intervals. $\mathrm{HR}, \mathrm{SaO}_{2}$ and capnography were monitored continuously.Throughout the procedure, all patients received $6 \mathrm{~L} / \mathrm{min}$ to $8 \mathrm{~L} / \mathrm{min}$ of $\mathrm{O}_{2}$ by mask to achieve pre-oxygenation in case of any respiratory compromise as a result of the anesthetic. Abdominal wall and chest excursions were also observed for respiratory effort.

A minimum of 4 medical staff were required for PhD NAPS ( 1 endoscopist, 1 nurse assisting the endoscopist, 1 nurse sedationist and 1 nurse at the head end of the bed monitoring the patient's airway). The nurse sedationist administered propofol and midazolam under the guidance of the endoscopist. All endoscopists had deep sedation privileges by the Accreditation and Privileges Committee of the hospital and had certification in Advanced Life Support (ALS). The endoscopists were also required to undergo an accreditation exercise on propofol seda-
Table 1 Nurse sedationist training program.

1. Three-month Nurse Sedationist Course (conducted by 'SA Health') includes ET intubation and laryngeal mask airway insertion

2. Nine-month Anaesthesia and Recovery course (conducted by 'SA Health')

3. Advanced Life Support (ALS) certification

4. Accreditation yearly by the Department of Anaesthesia which includes 5 laryngeal mask insertions in the theaters every 6 months

5. Six-month evaluation by an anesthetist observing a sedation list

6. 250 procedures/year to maintain accreditation

ET, endotracheal

tion by the Department of Anesthesia, with mandatory reaccreditation every 2 years. Each of the nurses had undergone a rigorous 1 -year training program ( $\triangleright$ Table 1 ). The nurse sedationist was responsible solely for drug administration and patient monitoring and did not have any other responsibility during the procedure. There were also monthly Sedation Committee meetings, which included the anesthetists, endoscopists, and nurse sedationists, where any problems that may have occurred would be deliberated and discussed.

Sedation was initiated with $1 \mathrm{mg}$ to $2 \mathrm{mg}$ of midazolam, followed by a slow induction bolus of $0.3-0 \mathrm{mg} / \mathrm{kg}$ to $75 \mathrm{mg} / \mathrm{kg}$ of propofol. There is no evidence to suggest that using propofol as a sole agent compared to in combination with another drug usually midazolam and/or fentanyl - decreases adverse events (AEs) or improves patient satisfaction [14-17]. In this study we used the combined approach (propofol and midazolam) to reduce the total dosage of propofol administration.Top-up doses of $10 \mathrm{mg}$ to $20 \mathrm{mg}$ of propofol were administered up to a maximum dose of $500 \mathrm{mg}$ with the aim of achieving moderate to deep sedation. Moderate to deep sedation is achieved when there are no spontaneous movements or phonation from the patient but vocal and tactile stimulation is still possible. There should be a sufficient depth and frequency of respiration without any evidence of desaturation or circulatory depression. The decision of when to administer further propofol was primarily made by the endoscopists, who verbally instructed the nurse as to when to give additional boluses. The indications for further doses were a rise in BP or spontaneous patient movement or phonation. If necessary, decisions to withhold sedation, increase oxygen flow or begin mask ventilation were made by the endoscopists, as were final decisions regarding the airway or management of a deteriorating patient.

The most common complications of sedation with propofol and midazolam are apneic episodes, acute desaturations, bradycardia, and hypotension. In the event of an apneic episode, propofol was ceased and basic airway maneuvers were initiated including head extension, jaw thrust and/or chin lift, and highflow oxygen delivered via a mask. Patients with acute desaturation were initially ventilated via a bag and mask. This was to be escalated to a laryngeal mask airway if an appropriate response was not achieved. Failing these measures, an urgent (expected response time of $1-2$ minutes) anesthetic review was to be requested. Bradycardia was to be managed with atropine boluses 
( $0.4 \mathrm{mg}$ up to a total dose of $2 \mathrm{mg}$ ). Hypotension was to be treated by simultaneously ceasing the propofol, placing the patient in the Trendelenburg position, and administering fluid boluses. If an adequate response was not obtained with these steps, ephedrine at a dose of $3 \mathrm{mg}$ to a max dose of $9 \mathrm{mg}$ was to be administered. If the patient had not responded to the above, the plan was to alert the duty anesthetist.

\section{Complications}

For the sake of this study, safety was considered to be no major complications or AEs requiring abortion of the procedure. The following were considered minor but significant intraoperative or postoperative complications: hypotension (systolic BP less than $100 \mathrm{mmHg}$ with a drop of greater than $20 \mathrm{mmHg}$ ), apnea (defined in this study as a reduction in airflow or respiratory effort for more than 10 seconds accompanied by a desaturation of $3 \%$ or more), hypoxia $\left(\mathrm{SaO}_{2}<90 \%\right)$, and bradycardia ( $\left.<50 \mathrm{bpm}\right)$. Major complications included death, persistently decreased conscious state, permanent injury, need for urgent anesthetic review, or need for hospitalization or endotracheal intubation. We considered ASA I and II patients as individuals with average anesthetic risk and ASA III patients as high risk. Procedure duration was defined as the time from the initial insertion of the endoscope to its withdrawal. Recovery time was defined as the time from endoscope withdrawal to recovery of full consciousness.

\section{Upper endoscopic/colonoscopic procedure and recovery}

Upper endoscopic/colonoscopic procedures were performed by gastroenterologists or surgeons, with upper endoscopies involving only simple diagnostic gastroscopies in this study. These procedures were performed using standard techniques and followed routine protocol. All patients remained in the endoscopy room until they regained consciousness, which was defined as the ability to maintain lucid conversation, with both the nurse and the gastroenterologist present. Patients were then moved to a recovery area, where a nurse monitored the patents' recovery. Before discharge, the recovery nurse rechecked patients' level of consciousness and ability to walk and ensured that procedural findings were communicated to them or their relatives.

\section{Results}

In total, 1000 patients were prospectively recruited for this study from January 2010 to October 2012. Nineteen patients were excluded from the study as they underwent sedation only with midazolam and fentanyl (due to a nurse sedationist being unavailable on the day of the procedure). Of the remainder, 345 were ASA 1 (50\% male), 620 were ASA II (44\% male) and 16 were ASA III ( $50 \%$ male). The sixteen patients classed as ASA III were reviewed by an anesthetist prior to their procedure date and deemed suitable for PhD NAPS. Indications for the procedures are summarized in $>$ Table 2 .

Demographic, baseline cardiorespiratory parameters and procedural factors are listed in $>$ Table 3 . The average total Pro-
- Table 2 Indications for endoscopy or colonoscopy or both.

\begin{tabular}{|l|c|}
\hline Endoscopy and colonoscopy & (\%) \\
\hline Abdominal pain & 2.3 \\
\hline Altered bowel habit & 4.2 \\
\hline Anaemia & 2.8 \\
\hline Barrett's & 1.5 \\
\hline Dyspepsia & 8.5 \\
\hline Dysphagia & 1.7 \\
\hline Inflammatory bowel disease & 1.4 \\
\hline Malabsorption & 0.8 \\
\hline Miscellaneous & 27.4 \\
\hline Per-rectal bleed & 7.7 \\
\hline Reflux & 19.9 \\
\hline Screening & 12.3 \\
\hline Surveillance & 5.3 \\
\hline Upper gastrointestinal bleed & 3.6 \\
\hline
\end{tabular}

pofol dose was $205.65 \mathrm{mg} \pm 92.66 \mathrm{mg}$. The average Midazolam dose was $1.11 \mathrm{mg} \pm 0.38 \mathrm{mg}$. The mean procedure duration was $19.20 \mathrm{~min} \pm 10.99 \mathrm{~min}$ and the mean recovery time was $23.44 \mathrm{~min} \pm 13.43 \mathrm{~min}$.

No major complications occurred. Minor cardiorespiratory complications occurred in $6.42 \%$ of patients (summarized in - Table 4), with 41 cases of hypotension (4.18\%), 22 cases of bradycardia (2.24\%) and 1 brief episode of apnea in a woman who was also hypotensive. All cases of hypotension responded to IV fluid boluses and required no further management. The lowest recorded systolic blood pressure was $75 \mathrm{mmHg}$. All patients with bradycardia improved spontaneously and treatment with atropine was not required. The lowest recorded heart rate was 38 bpm. The brief episode of apnea resolved spontaneously without requiring any respiratory intervention. There were no episodes of hypoxia. No patients required anesthetist review during the procedure or immediately postoperatively.

\section{Discussion}

PhD NAPS is increasingly being used worldwide in low-risk patients undergoing endoscopy and/or colonoscopy because of recognition of its safety, rapid and predictable induction and recovery times, earlier patient discharge, increased patient and endoscopist satisfaction, and its presumed economic advantage [2-5]. This study adds to the mounting evidence that PhD NAPS is safe when stringent patient selection is used and those administering the sedative agents have received the appropriate training [6-9]. Complications encountered were all minor and predictable based on the nature of the procedure and the side effect profile of the medications utilized, and easily managed by the nurse sedationist and endoscopist. In keeping with our definition of safe, there were no major complica- 
- Table 3 Demographic, baseline cardiorespiratory parameters and procedural factors.

\begin{tabular}{|c|c|c|c|c|}
\hline Variables & $\begin{array}{l}\text { ASA I } \\
(n=345)\end{array}$ & $\begin{array}{l}\text { ASA II } \\
(n=620)\end{array}$ & $\begin{array}{l}\text { ASA III } \\
(n=16)\end{array}$ & $\begin{array}{l}\text { Total } \\
(n=981)\end{array}$ \\
\hline Age & $45.49 \pm 14.95$ & $57.232 \pm 13.93$ & $65.063 \pm 11.29$ & $53.23 \pm 15.38$ \\
\hline Men & $173(50.14 \%)$ & $270(43.55 \%)$ & $8(50.00 \%)$ & 451 (45.97\%) \\
\hline Pre-SBP & $126.51 \pm 15.30$ & $132.33 \pm 18.17$ & $136.33 \pm 23.47$ & $130.35 \pm 17.52$ \\
\hline Pre-DBP & $79.98 \pm 11.26$ & $80.75 \pm 11.73$ & $76.73 \pm 14.20$ & $80.41 \pm 11.61$ \\
\hline Pre-PR & $74.12 \pm 12.76$ & $76.77 \pm 14.48$ & $70.21 \pm 16.06$ & $75.74 \pm 13.98$ \\
\hline Pre-RR & $18.51 \pm 1.75$ & $18.43 \pm 1.70$ & $18.80 \pm 1.61$ & $18.47 \pm 1.71$ \\
\hline Pre-SaO ${ }_{2}$ & $99.06 \pm 1.24$ & $98.34 \pm 1.74$ & $98.33 \pm 1.35$ & $98.59 \pm 1.61$ \\
\hline Propofol dose & $208.35 \pm 84.335$ & $205.02 \pm 97.65$ & $171.88 \pm 53.32$ & $205.65 \pm 92.66$ \\
\hline Midazolam dose & $1.16 \pm 0.43$ & $1.09 \pm 0.35$ & $0.97 \pm 0.29$ & $1.11 \pm 0.38$ \\
\hline Intra-SBP & $122.12 \pm 15.05$ & $127.49 \pm 18.51$ & $128.50 \pm 16.90$ & $125.61 \pm 17.52$ \\
\hline Intra-DBP & $71.50 \pm 11.91$ & $73.29 \pm 13.34$ & $69.88 \pm 13.67$ & $72.60 \pm 12.88$ \\
\hline Intra-PR & $75.09 \pm 12.06$ & $74.55 \pm 12.30$ & $69.38 \pm 14.82$ & $74.66 \pm 12.27$ \\
\hline Intra-RR & $19.42 \pm 0.98$ & $18.94 \pm 1.72$ & $20.00 \pm 0.00$ & $19.14 \pm 1.48$ \\
\hline Intra-SaO ${ }_{2}$ & $98.65 \pm 1.17$ & $98.44 \pm 1.28$ & $98.19 \pm 1.28$ & $98.51 \pm 1.25$ \\
\hline Procedure duration & $17.54 \pm 10.32$ & $20.16 \pm 11.29$ & $17.88 \pm 9.37$ & $19.20 \pm 10.99$ \\
\hline Post-SBP & $116.79 \pm 13.57$ & $121.59 \pm 16.39$ & $126.81 \pm 18.59$ & $119.99 \pm 15.67$ \\
\hline Post-DBP & $71.35 \pm 11.66$ & $72.60 \pm 11.19$ & $71.19 \pm 13.01$ & $72.14 \pm 11.39$ \\
\hline Post-PR & $70.71 \pm 12.01$ & $70.05 \pm 12.00$ & $66.44 \pm 11.97$ & $70.22 \pm 12.00$ \\
\hline Post-RR & $19.08 \pm 1.83$ & $18.95 \pm 1.55$ & $19.5 \pm 1.24$ & $19.00 \pm 1.65$ \\
\hline Post- $\mathrm{SaO}_{2}$ & $98.99 \pm 1.40$ & $98.78 \pm 1.40$ & $98.56 \pm 1.67$ & $98.85 \pm 1.41$ \\
\hline Recovery duration & $24.01 \pm 13.89$ & $23.11 \pm 13.22$ & $23.93 \pm 11.39$ & $23.44 \pm 13.43$ \\
\hline
\end{tabular}

ASA, American Society of Anesthesiologists; SBP, systolic blood pressure; DBP, diastolic blood pressure; PR, pulse rate; RR, respiratory rate; $\mathrm{Sa}_{2}$, oxygen saturation

- Table4 Cardiorespiratory complication rates.

\begin{tabular}{|c|c|c|c|c|c|c|c|}
\hline Outcome & Total & $\begin{array}{l}\text { Rate } \\
\text { (\%) }\end{array}$ & Endoscopy & Colonoscopy & $\begin{array}{l}\text { Endoscopyl } \\
\text { colonoscopy }\end{array}$ & Intervention & No intervention \\
\hline Intraoperative hypotension & 16 & 1.63 & 4 & 9 & 3 & 4 & 12 \\
\hline Postoperative hypotension & 25 & 2.55 & 11 & 13 & 1 & 9 & 16 \\
\hline Total hypotension & 41 & 4.18 & 15 & 22 & 4 & 13 & 28 \\
\hline Intraoperative bradycardia & 12 & 1.22 & 5 & 6 & 1 & 7 & 5 \\
\hline Postoperative bradycardia & 10 & 1.02 & 3 & 7 & 0 & 4 & 6 \\
\hline Total bradycardia & 22 & 2.24 & 8 & 13 & 1 & 11 & 11 \\
\hline All patients & 981 & & 440 & 420 & 121 & 309 & 672 \\
\hline
\end{tabular}

tions and no procedures were aborted. The study was conducted at a single tertiary teaching hospital and excluded patients with a body mass index above $35 \mathrm{~kg} / \mathrm{m}^{2}$. While this may limit the generalizability of the results, these patients would be considered high risk and in any setting would require anesthetistdirected sedation.
Despite these outlined advantages, this method of sedation is not universally employed due to concerns about its safety. In 2009, following a review of twenty-eight papers on the topic, the American Society for Gastrointestinal Endoscopy issued a position statement with regard to non-anesthetist administration of Propofol [5]. In this statement, the society acknowledged the safety of PhD NAPS, provided that it was delivered 
by a specifically trained team of individuals. Shortly thereafter, in 2010, the European Society of Gastrointestinal Endoscopy, combined with the European Society of Gastroenterology and Endoscopy Nurses and Associates, and the European Society on Anaesthesiology (ESA), released a set of guidelines for nonanesthetists administering Propofol for gastrointestinal endoscopy [4]. The aim of these consensus-based guidelines was to provide non-anesthetists with a comprehensive framework for propofol sedation during endoscopy, recognizing PhD NAPS as a safe sedation method in gastrointestinal procedures [4]. However, the European Journal of Anaesthesiology then released a 2011 consensus statement raising theoretical safety concerns regarding the known adverse cardiopulmonary effects of propofol [18]. Subsequently, the ESA officially retracted their endorsement of PhD NAPS. While there are theoretical risks in any procedure, the acceptance of PhD NAPS should be based on the increasing amounts of high-level evidence regarding its efficacy and safety.

Numerous studies conducted worldwide have supported the safety of PhD NAPS. Rex et al (2009) retrospectively studied 646080 cases in which PhD NAPS was used, and found $0.1 \%$ of patients needed bag and mask ventilation, 11 patients required intubation, and 4 patients died [10]. All 4 deaths were in patients who had severe premorbid illness with ASA scores of III and IV, and therefore would not have fulfilled our stringent selection criteria. In 3 of the 4 cases, treatment was withdrawn at the request of family members or carers, and in the fourth case death resulted from metastatic pancreatic cancer causing duodenal erosion with an arterial bleed [10]. A cost-analysis showed that if an anesthetist was present at each case, and was able to prevent the death of all 4 of these patients, the cost per life year saved would have been approximately US $\$ 2.6$ million [10].

Two important comparisons in this discussion are PhD NAPS versus anesthetist-delivered sedation, and the currently accepted practice of endoscopist-delivered opioid and benzodiazepine sedation versus propofol. In their 2015 meta-analysis, which included only prospective trials, Goudra et al found equal rates of hypoxia, and lower rates of airway intervention in the PhD NAPS group versus the anesthetist-directed propofol sedation [11]. Furthermore, PhD NAPS has been shown to be at least as safe as the currently accepted practice of conscious sedation with opioid and benzodiazepine. A 2005 meta-analysis found no increase in cardiopulmonary AEs when using propofol sedation versus traditional opioid/benzodiazepine sedation for endoscopy, and possibly a slight decrease in cardiopulmonary AEs during colonoscopy [12]. Anesthetic services are in high demand and costly. As an alternative to anesthetist-directed sedation, nurse sedationists and endoscopists with sedation privileges can be trained to safely deliver sedation during gastrointestinal procedures. Although there is a presumed economic benefit to not requiring an anesthetist for endoscopic procedures, this requires substantiation by a formal cost analysis.

\section{Conclusion}

In conclusion, PhD NAPS is safe in endoscopy and colonoscopy when the endoscopist and nursing staff involved are appropriately trained and strict patient selection criteria are employed.

\section{Competing interests}

None

References

[1] Mackenzie N, Grant IS. Propofol for intravenous sedation. Anesthesia 1987; 42: 3-6

[2] McQuaid KR, Laine L. A systematic review and meta-analysis of randomized, controlled trials of moderate sedation for routine endoscopic procedures. Gastrointest Endosc 2008; 67: 910- 923

[3] Deenadayalu VP, Eid EF, Goff JS et al. Non-anesthesiologist Administered Propofol Sedation for Endoscopic Procedures: A Worldwide Safety Review. Gastrointest Endosc 2008; 67: AB107

[4] Dumonceau JM, Riphaus A, Aparicio JR et al. European Society of Gastrointestinal Endoscopy, European Society of Gastroenterology and Endoscopy Nurses and Associates, and the European Society of Anaesthesiology Guideline: Non-anesthesiologist administration of propofol for Gl endoscopy. Endoscopy 2010; 42: 960 - 974

[5] Vargo J], Cohen LB, Rex DK et al. Position statement: Nonanesthesiologist administration of propofol for Gl endoscopy. Gastroenterology 2009; 137: $2161-2167$

[6] Daneshmend TK, Bell GD, Logan RF. Sedation for upper gastrointestinal endoscopy: results of a nationwide survey. Gut 1991; 32: 12 - 15

[7] Froehlich F, Gonvers J], Fried M. Conscious sedation, clinically relevant complications and monitoring of endoscopy: results of a nationwide survey in Switzerland. Endoscopy 1994; 26: $231-234$

[8] Quine MA, Bell GD, McCloy RF et al. Prospective audit of upper gastrointestinal endoscopy in two regions of England: safety, staffing, and sedation methods. Gut 1995; 36: $462-467$

[9] Thompson AM, Wright DJ, Murray W et al. Analysis of 153 deaths after upper gastrointestinal endoscopy: room for improvement. Surg Endosc 2004; 18: $22-25.15$

[10] Rex DK, Deenadayalu VP, Eid E et al. Endoscopist-Directed Administration of Propofol: A Worldwide Safety Experience. Ygast 2009; 137: $1229-1237$

[11] Goudra B, Singh PM, Gouda G et al. Safety of Non-anesthesia Provider-Administered Propofol (NAAP) Sedation in Advanced Gastrointestinal Endoscopic Procedures: Comparative Meta-Analysis of Pooled Results. Dig Dis Sci 2015; 60: 2612-2627

[12] Qadeer MA, Vargo JJ, Khandwala F et al. Propofol Versus Traditional Sedative Agents for Gastrointestinal Endoscopy: A Meta-analysis. Clin Gastroenterol Hepatol 2005; 3: 1049-1056

[13] American Society of Anesthesiologists. Statement on safe use of propofol. ASA House of Delagates, Illanois, USA: 2014

[14] Ong WC, Santosh D, Lakhtakia S et al. A randomized controlled trial on use of propofol alone versus propofol with midazolam, ketamine, and pentazocine "sedato-analgesic cocktail" for sedation during ERCP. Endoscopy 2007; 39: 807-812

[15] Padmanabhan U, Leslie K, Eer AS et al. Early cognitive impairment after sedation for colonoscopy: the effect of adding midazolam and/ or fentanyl to propofol. Anesthesia and Analgesia 2009; 109: 1448 1455 
[16] Paspatis GA, Manolaraki MM, Vardas E et al. Deep sedation for endoscopic retrograde cholangiopancreatography: intravenous propofol alone versus intravenous propofol with oral midazolam premedication. Endoscopy 2008; 40: 308-313

[17] VanNatta ME, Rex DK. Propofol alone titrated to deep sedation versus propofol in combination with opioids and/or benzodiazepines and ti- trated to moderate sedation for colonoscopy. Am J Gastroenterol 2006; 101: 2209-2217

[18] Perel A. Non-anaesthesiologists should not be allowed to administer propofol for procedural sedation: a Consensus Statementof 21 European National Societies of Anesthesia. Eur J Anaesthesiol 2011; 28: $580-584$ 